\title{
Perspectivas teóricas para la Educación Ambiental en básica secundaria. Teoría y práctica ${ }^{1}$
}

Liliana Haydé Gutiérrez Sabogal²

IED Ignacio Pescador, Choachí, Colombia

jomapagu43@hotmail.com

2 Docente de Ciencias Naturales de la IED Ignacio Pescador, Choachí - Cundinamarca. Líder del grupo de investigación Gototas de agua. 


\title{
Perspectivas teóricas para la Educación Ambiental en básica secundaria. Teoría y práctica
}

\section{Resumen:}

El presente artículo expone los antecedentes y conclusiones de una propuesta en Educación Ambiental —EAsustentada en referentes teóricos que pueden enmarcar procesos de enseñanza y aprendizaje en esta disciplina en básica secundaria, todo ello después de la revisión y el análisis riguroso de la didáctica en educación complementada con la experiencia profesional docente. Para poner en escena este marco conceptual se plantean y desarrollan actividades dentro del Proyecto Ambiental Escolar - PRAE - de la IED Ignacio Pescador como una manera de poner en práctica la teoría analizada. De este modo se logran resultados que validan el marco de referencia propuesto para producir conocimiento sobre la teoría didáctica -la cual va dirigida a formadores profesionales docentes que acompañarán el proceso de enseñanza de la EA en las instituciones educativas de básica secundaria - y el proceso de construcción de conocimiento en el alumnado.

Palabras clave: Educación ambiental, paradigma de la complejidad, modo 3 de producción de conocimiento, constructivismo, proyecto ambiental escolar.

\section{Theoretical perspectives for the Environmental Education in high school. Theory and practice}

\begin{abstract}
This article exposes the background and conclusions of an Environmental Education purpose -EE- supported by theoretical models able to frame processes of teaching and learning applied to EE in the high school. All of this come from strict revision and analysis of complemented educational didactics, through the professional teaching experience. To reveal this conceptual framework, the activities are posed and developed within the Environmental School Project - ESP - of the District Education Institute - DEI - Ignacio Pescador as a mode to put into practice the analyzed theory. Thus, the results obtained validate the referential framework proposed to produce knowledge about the instructive theory - which is aimed to professional teachers who will be added to the teaching process of EE into the high school institutes - and the process of knowledge creation in the student body.
\end{abstract}

Key words: Environmental education, complexity paradigm, mode 3 of knowledge production, constructivism, environmental school project.

\section{Perspectivas teóricas para a educação ambiental na base secundária. Teoria e prática}

\section{Resumo}

Este artigo apresenta os antecedentes e os resultados de uma proposta de Educação Ambiental -EA- apoiada por referências teóricas que pode enquadrar os processos de ensino e aprendizagem nesta disciplina na base secundária, tudo isso após da revisão e a análise rigorosa da didática na educação suplementada com experiência de ensino. Para encenar essa estrutura conceptual foi proposto e desenvolvido actividades no âmbito do Projecto Ambiental Escola -PRAE- no IED Ignacio Pescador como um jeito de implementar a teoria analisada. Deste modo conseguem-se resultados que validam o marco de referência proposto para produzir conhecimento sobre a teoria didática - a qual vai dirigida a formadores profissionais docentes que acompanharão o processo de ensino da EA nas instituições educativas de básica secundária- e o processo de construção de conhecimento no alumnado.

Palavras-chave: Educação ambiental, paradigma da complexidade, modo 3 de produção de conhecimento, constructivismo, projeto ambiental escolar. 


\section{Introducción}

La educación ambiental —EA- en las escuelas colombianas se ha convertido en un verdadero reto para las instituciones educativas ya que el ciudadano actual requiere de competencias ciudadanas estrechamente relacionadas con el ambiente y, además, la solución de problemáticas socioambientales inmersas en de la cotidianidad de sus actividades. De ahí la importancia de una propuesta acerca de los pilares del marco teórico bajo los cuales se pueden desarrollar procesos de enseñanza y aprendizaje de la EA que se constituya como un aporte a la educación y a la sociedad para solucionar problemáticas socioambientales desde la sostenibilidad, la búsqueda de la solidaridad, la paz y el manejo de conflictos de forma pacífica.

Tener claros los paradigmas que pueden sostener el desarrollo de procesos de aprendizaje y enseñanza de una EA que responda a las necesidades actuales de la sociedad es un punto de partida para que la escuela se convierta en el eje central de la formación de ciudadanos y ciudadanas que sean capaces de tomar decisiones de forma consciente e informada y actúen de acuerdo a la compleja comprensión de las problemáticas que debe resolver para mejorar tanto su nivel de vida como el de todas las demás formas de vida.

La continua reflexión sobre las acciones pedagógicas que se realizan cotidianamente en la escuela permitió realizar un marco teórico, desde diversas perspectivas, alrededor del cual se cree que la EA puede alcanzar sus objetivos desde la educación y sus expectativas. Una vez desarrolladas estas perspectivas se planteó y desarrolló una propuesta interdisciplinaria y transversal denominada: «Reconociendo nuestro territorio chiguano», comprendida dentro del Proyecto Ambiental Escolar — PRAE— de la IED Ignacio Pescador.

El objetivo de este artículo es exponer las posibles perspectivas que pueden enmarcar los procesos de enseñanza y aprendizaje de la EA y socializar una puesta en práctica de este marco conceptual en la IED Ignacio Pescador, ubicada en el municipio de Choachí - Cundinamarca, a través del PRAE.

\section{Antecedentes}

El trabajo realizado se ha llevado basándose en una reflexión del área de ciencias naturales y educación ambiental de la IED Ignacio Pescador. Creemos que en esta institución la Educación Ambiental ha sido un poco olvidada desde el punto de vista de la formación de los estudiantes puesto que, a pesar del sinnúmero de actividades ecológicas que se realizan dentro de la escuela, no se han logrado cambios en los comportamientos de la comunidad. 
Es así como se plantea en primera instancia un análisis teórico de la cuestión, desde diferentes autores, que se complementa con la experiencia docente para planear posteriormente acciones pedagógicas a través del PRAE de las instituciones.

\section{Perspectivas que sustentan la propuesta}

La propuesta para el trabajo pedagógico en EA en básica secundaria se puede resumir en cuatro perspectivas que señalan los caminos y reflexiones metodológicas así como la comprensión y análisis de las problemáticas ambientales que atañen a la EA; tales son: a) perspectiva crítica de la enseñanza, b) perspectiva constructivista de la construcción del conocimiento, c) perspectiva de la complejidad y, por último, d) perspectiva modo 3 de producción de conocimiento. Cada una de estas perspectivas se desarrollará a continuación mediante un análisis de lo que ha venido sucediendo a través de la historia para dilucidar el porqué de estas bases teóricas dentro de una investigación cuyo interés se enmarca en los procesos de aprendizaje y enseñanza de la Educación Ambiental.

\section{a) Perspectiva crítica de la enseñanza}

La visión positivista que se generalizó a lo largo de los años se ha caracterizado por la objetividad en el conocimiento. Tal perspectiva reconoce verdades que se deben transmitir en el proceso de enseñanza para la interpretación de la realidad social por medio de la separación entre los investigadores educativos, los diseñadores de la política curricular y los practicantes de la profesión docente. El positivismo así entendido deja a los practicantes la mera posibilidad de impartir «paquetes» curriculares — que imponen los diseñadores de acuerdo a requerimientos generados por los investigadores por su especialización en disciplinas e interés por problemas estrechos - que genera una pérdida de la complejidad de la realidad al asumir la escuela y la estructura de la sociedad como algo «dado». Estas situaciones privan de crítica al proceso de enseñanza de acuerdo a lo que exponen Carr y Kemmis (citado de Kember y Lingow, 1988).

A diferencia de esa concepción, siguiendo a autores como García Pérez (s.f.), García (1998), Arroyo (2012) y Fernández (2012), en esta investigación se asume una visión dialéctica de la racionalidad que intenta, en primera instancia, mediante procesos subjetivos y objetivos, comprender los límites de las situaciones y cómo podrían cambiar; segundo, desarrollar una investigación para la educación comprometida con procesos participativos y colaborativos de autorreflexión; tercero, producir sistemáticamente conocimiento dentro de una comunidad autocritica de participantes y, finalmente, buscar la unidad entre la teoría y la práctica educativa en la que el profesional docente asuma el rol de investigador y diseñador del currículo en un intento por transformar el proceso de la enseñanza. 
La perspectiva crítica sostenida está inmersa en el rompimiento de tensiones que de una u otra forma inciden en el proceso de enseñanza tradicionalista que invade nuestra escuela:

1. Dicotomía entre la práctica y la teoría: la segunda fórmula y justifica principios que declaran lo que ha de hacerse en la práctica.

2. Dicotomía entre investigador y profesional docente: el primero impone los principios que el profesor necesita para solucionar sus problemas en el aula.

3. Dicotomía entre los diferentes tipos de conocimiento: el conocimiento cotidiano, escolar y científico se consideran diferentes, antagónicos y sin posibilidades de encontrarse, reestructurarse y enriquecerse en la escuela. La misma situación ocurre entre el conocimiento profesional docente teórico y el conocimiento producido por investigadores externos.

4. Dicotomía entre especialización y generalización: la fragmentación del conocimiento en especialidades no permiten la unificación, la interdisciplinariedad y la transversalidad para la comprensión y solución de problemáticas.

El rompimiento de estas tensiones mediante la reflexión crítica y responsable sobre el proceso de enseñanza involucra cambios de paradigmas que son necesarios para que la EA cumpla con sus retos, esto es, un cambio de comportamientos que lleven a una acción informada y coherente con la situación ambiental y logre transformar situaciones de acuerdo a lo que requiere la sociedad. En otras palabras, las contradicciones ahora se pueden superar, no se debe optar por uno de los polos, sino que el análisis y la comprensión genera conocimiento que puede sintetizar y generar opciones para trascender el estado de las cosas contradictorias; entonces los elementos, antes considerados separados y diferentes, se convierten en mutuamente constitutivos e implican la posibilidad de alcanzar nuevas posibilidades.

Esta perspectiva crítica en la investigación implica que:

- Los problemas socioambientales se pueden transformar mediante la EA: se analizan y comprenden desde lo local, teniendo en cuenta las interrelaciones implícitas y explicitas que, mediante la interdisciplinariedad y transversalidad, intentan unificar la marcada fragmentación del conocimiento para tener una visión más compleja de la realidad y, por tanto, soluciones eficientes y exitosas.

- Los actores del proceso educativo son participantes en la situación estudiada, coinvestigadores, colaboradores y coproductores de conocimiento para lograr así mejores prácticas. 
- La intervención en la situación problema va más allá del desarrollo de teorías o interpretaciones para implantar cambios que ayuden al progreso de la teoría o al entendimiento de ella.

- El desarrollo profesional docente se evidencia en la comprensión autónoma de los principios educacionales básicos y no en preocupaciones de orden instrumental o utilitario que pretenden mantener su papel hegemónico en la educación. Se deja atrás la creencia de que el profesional docente es mejor si utiliza cada vez más hábilmente un fondo común de conocimientos pedagógicos ya establecidos.

- El profesional docente produce conocimiento teórico desde la cotidianidad para comprender la realidad y transformar la situación-problema en la búsqueda de su solución.

- El profesor rehace las condiciones de acción informada y somete a revisión permanente tanto la acción como los conocimientos que la informan en una búsqueda continua del obrar correcta y justificadamente, lo cual mejora la práctica docente.

La perspectiva crítica aquí descrita justifica el uso de la metodología de investigación-acción como una alternativa para el desarrollo profesional docente por medio de ciclos de planeación, acción, observación y reflexión mediante un conocimiento que se genera en ciclos de acción-reflexión importante para el ejercicio de la EA. Un error de tal ejercicio ha sido el haber sido asumido desde diversas profesiones y considerar que cualquier persona puede desarrollarlo haciendo que los procesos de enseñanza se mantengan al margen del rigor pedagógico y didáctico requeridos para garantizar su calidad y su efectividad.

\section{b) Perspectiva constructivista de la construcción del conocimiento}

El constructivismo, desde la mirada de algunos autores como Cubero (2000), ha sido una de las perspectivas que sirven de marco a la EA. La Educación Ambiental, como cualquier tipo de educación, necesita develar el papel de cada actor en el proceso y el estudio de la didáctica para buscar las mejores alternativas y de ese modo lograr los objetivos propuestos. La mirada tradicional positivista, que ha sido hegemónica en la escuela desde sus inicios, debe ser analizada para lograr su comprensión y las alternativas de solución más eficientes de acuerdo a los requerimientos locales, sin descuidar los efectos globales.

Cubero, referenciada por Fernández (2012), afirma que:

El constructivismo es una herramienta de conocimiento que no puede reducirse a una teoría o a un único planteamiento metodológico. Muchas teorías pueden integrarse dentro de la perspectiva constructivista y cada una de ellas ofrece diferentes modos de interpretar la naturaleza del conocimiento y los procesos 
que lo producen. Por lo tanto, existen diferentes posiciones constructivistas desde las que elaborar propuestas educativas. Cada una de ellas hace más hincapié en algunos elementos que en otros (p. 61).

Los tres principios fundamentales del constructivismo, que a la vez pueden ser los pilares teóricos bajo los cuales se desarrollan los procesos de enseñanza y aprendizaje de EA, han sido explicados por Cubero: «una epistemología relativista, una concepción de la persona como agente activo, y una interpretación de la construcción del conocimiento como un proceso interactivo situado en un contexto cultural e histórico» (García y Cano, 2006). Lo anterior supone «situaciones de aprendizaje en las que haya contraste de ideas y negociación democrática del conocimiento» que movilicen el conjunto de las creencias de la persona y que tengan afinidad y continuidad con su experiencia cotidiana:

- Epistemología relativista: los contenidos son entendidos como realidades relativas, se negocian entre los participantes en el proceso de construcción y de esa guisa se construyen versiones de los hechos. Las realidades relativas se justifican por medio de la argumentación, la búsqueda de consenso, las analogías, el recurso a la evidencia perceptiva y la autoridad de los especialistas como fuente de conocimiento. El carácter relativo y dinámico implica poner por delante al proceso de aprendizaje en sí frente al resultado final, entendiéndose éste como una formulación única y cerrada del contenido que se está trabajando.

- Concepción de la persona como agente activo: la visión constructivista muestra que la persona que aprende se convierte en un agente activo cuando asume un papel protagonista gracias al crecimiento de la autonomía y control de su aprendizaje. Requiere potenciar la conciencia, el control y la reflexión de los alumnos sobre su propio proceso de aprendizaje aprender a aprender-.

- Interpretación de la construcción del conocimiento como un proceso interactivo y situado en un contexto cultural e histórico:

- Construcción cultural del conocimiento porque es un producto social construido por comunidades diferentes y acumulado a través de la historia. La interacción se da en un contexto de prácticas culturalmente organizadas y con herramientas y contenidos culturales.

- Construcción social del conocimiento ya que todo conocimiento se construye en un ambiente que le da sentido y que forma parte de los procesos de construcción —escenario sociocultural-.

- Aprendizaje significativo como elemento esencial de la concepción constructivista del aprendizaje escolar:

Aquel que las personas desarrollan cuando son capaces de otorgar un significado al 
contenido objeto de aprendizaje, mediante la actualización de aquellos esquemas de conocimiento que se ponen en juego dependiendo de la situación de que se trate. [...] [P]ara comprender es necesario traducir algo a las propias ideas o palabras y aprender significados supone un cambio en las ideas como consecuencia de su interacción con la nueva información (Pozo, 1996; Fernández, 2012, p. 76).

- Cambios conceptuales en el aprendizaje:

El proceso de asimilación-acomodación se produce al confrontar las representaciones que tenemos sobre la realidad con los resultados de las actuaciones que realizamos sobre ella. La realidad, por medio de su resistencia — física o social- activa el conocimiento, ya que son necesarias nuevas explicaciones que resuelvan los problemas que dicha realidad nos plantea. [...] [E]s necesaria la modificación o reorganización de nuestro sistema de ideas; es decir, el cambio conceptual. El nivel de construcción depende del tipo de relación —entre la nueva información y el conocimiento previo-, del grado de conciencia y de la reflexión que hagamos sobre dicha relación (Fernández, 2012, pp. 77-78).

- Funcionalidad y transferencia en los aprendizajes:

La posibilidad de aplicar lo que se conoce de modo significativo supone que las personas pueden establecer un mayor y más profundo conjunto de relaciones entre los contenidos objeto de estudio y ofrece la posibilidad de establecer un marco de conocimientos más comprensivo y de mayor significación para los conocimientos y actividades nuevas que se emprenden (Fernández, 2012, p. 81).

Ahora bien, si relacionamos los objetivos de la EA con el método didáctico podemos retomar la siguiente reflexión de García y Cano (2006): «si lo que queremos es un cambio radical del pensamiento y de la conducta de las personas, es decir, un cambio lento, gradual, difícil, a contracorriente de la cultura dominante, tenemos que pensar que tal cambio sólo se puede lograr con estrategias de corte constructivista» (p. 119). De ahí la consideración del constructivismo como marco de referencia para llevar a cabo la estrategia didáctica que pretende ser el comienzo de este trabajo investigativo.

Es así como cobra sentido la siguiente explicación que se hace del conocimiento escolar:

Enriquecer el conocimiento cotidiano de los sujetos con una visión más compleja del mundo, trabajando en el aula un tipo de conocimiento — el conocimiento escolarque, originado en la integración didáctica de diferentes formas del saber — científico, ideológico, cotidiano, artístico, etc.- - suponga una reconstrucción critica y una mejora del conocimiento cotidiano que capacite a los individuos para una participación más consciente en la gestión y resolución de problemas socioambientales propios de nuestro mundo. (González, 2011, p. 17; García, et al., 1997).

La propuesta del modelo de metodología, que García (1998) refiere como metodología basada en la investigación del alumno, es una posibilidad para lograr 
procesos de enseñanza y aprendizaje con la construcción de conocimientos en el alumnado que logren cambios en los comportamientos y acciones ciudadanas, objetivos de la EA.

\section{c) Perspectiva de la complejidad}

Cuando de EA se trata son decisivas las interrelaciones que se tejen entre las diferentes esferas de la sociedad: económica, política, cultural, social y educativa; pero asimismo entre las diferentes disciplinas del saber: ciencias naturales, ciencias sociales, ecología, antropología, meteorología y otras directa o indirectamente relacionadas. Por esto es importante analizar el paradigma de la complejidad y compararlo con el paradigma simplificador en el cual actualmente se desarrolla la EA en nuestras escuelas.

El paradigma simplificador no puede satisfacer la necesidad de comprensión de realidades y problemas socioambientales pues la realidad es multidimensional, es organización compleja en la que distintos seres se entremezclan e interfieren en interacciones complementarias, concurrentes, antagónicas e inciertas. La epistemología de la complejidad, desarrollada por Edgar Morin, reformula y supera estas limitaciones:

Pero la diferencia aquí es precisamente de paradigma, no se trata ya de obedecer a un principio de orden - excluyendo el desorden—, de claridad — excluyendo lo oscuro-, de distinción —excluyendo las adherencias, participación y comunicaciones-, de disyunción - excluyendo el sujeto, la antinomia, la complejidad-, es decir, un principio que una la ciencia a la simplificación lógica. Se trata, por el contrario, a partir de un principio de complejidad, de unir lo que estaba disjunto (como se cita en García, 1998, p. 84).

El paradigma de la complejidad, como lo enuncia M. García (2010), representa una actitud abierta, antirreducionista y relativizadora que se contrapone al dogmatismo y al uso de recetas simplicistas; supone la existencias del azar, de las incertidumbres, las paradojas y contradicciones; busca nuevas maneras de formular soluciones a los problemas, más que nuevas verdades que expliquen la realidad. Supone, entonces, el análisis de problemas en contexto, cuya comprensión requiere de lo aleatorio y lo relacional.

La complejidad no establece dicotomías sino complementariedad entre el conocimiento de la parte y el todo, el análisis y la síntesis, el conocimiento científico y el filosófico e ideológico, la teoría y la práctica, el orden y el caos, lo natural y lo social, el conocimiento disciplinar y el interdisciplinar, las especialidades y la generalización, el conocimiento individual y social del conocimiento. Sin embargo, la complementariedad no excluye el antagonismo, sino que lo integra generando la participación de una misma totalidad. 
La perspectiva de la complejidad se acerca a la concepción de la naturaleza como un sistema en constante equilibrio y evolución, que integra a la humanidad, según Fernández (2012), «como un nudo en la red de los sistemas de los que forma parte» dejando atrás la mirada antropocentrista —que ha legado huellas imborrables y destrucción del planeta - para integrar conceptos como biocentrismo y ambiocentrismo, más afines con el desarrollo sostenible que se quiere implementar según las conferencias y acuerdos mundiales.

De igual forma, Fernández (2012) muestra cómo el paradigma de la complejidad reorienta la acción ciudadana hacia un modelo de vida que entiende la libertad como responsabilidad, la convivencia como democracia participativa - en una sociedad en permanente construcción - y la actuación comunitaria como proyección global en la que lo local y lo global se pueden complementar. En otras palabras, el paradigma de la complejidad:

Capacita al ciudadano para que coloque en los primeros lugares de esta escala valores que permitan construir un mundo mejor para todos. Por tanto, el paradigma de la complejidad nos hace tomar conciencia y nos ayuda a la hora de lograr un cambio. En definitiva, nos capacita para un cambio, ya que sin reconocer la necesidad de un cambio, éste no se llevará a cabo y no actuaremos en consecuencia éticamente en nuestro planeta (Fernández, 2012, p. 46).

Todo lo anterior evidencia cómo la perspectiva de la complejidad es un pilar para desarrollar la investigación de los procesos de enseñanza y aprendizaje de la EA, que como tal requiere no solamente de una perspectiva crítica y constructivista sino de una compleja, desde los diferentes enfoques que permiten la comprensión de la realidad.

\section{d) Perspectiva modo 3 de producción de conocimiento}

El modo 3 de producción de conocimiento, según Acosta (2013), es una propuesta sobre una forma de construir conocimiento dentro de un marco alternativo que pretende dar indicios para el trabajo investigativo y social en ámbitos como la EA. Esta propuesta exige procesos de producción de conocimiento que respondan a características diferentes a las concebidas en los modos 1 y 2 . La crisis de la Modernidad —Modernidad que no pudo dar solución a las problemáticas socioambientales sino que generó mayores desequilibrios ecológicos planetariosy la posterior reflexión mundial, que inició con la Conferencia de las Naciones Unidas sobre el Medio Ambiente en Estocolmo en 1972, exigen la comprensión y superación de problemáticas socioambientales que evidencian la importancia que tiene para la conservación del planeta los procesos de producción de conocimiento responsables con las necesidades de la sociedad.

El modo 3 de conocimiento, con sus características, satisface las necesidades y retos de la Educación Ambiental porque: 
- El conocimiento pasa de las manos de aquellos que pueden financiar su producción a manos de la humanidad, es decir, deben sumarse las demandas de los actores de la cuarta y quinta hélice de Carayannis (2012): la sociedad civil y el ambiente — no solamente los intereses gubernamentales, económicos y educativos - para que así aporten a la solución de las grandes problemas de la humanidad que involucran a los grupos más vulnerables.

- La problematización de la situación socioambiental es un proceso que involucra a todos los actores del tejido social en el que el flujo de información empieza en la base de la sociedad en un proceso button up - que incluye la interrelación de las diversas variables posibles que afectan el problema-.

- El modo 3 logra hacer síntesis de saberes de distintos tipos: cotidianos, escolares, de sentido común, metadisciplinares y científicos, que pasan por etapas de contradicción pero que maduran en procesos de síntesis y logran convertirse en elementos mutuamente constitutivos y posibilitan así nuevas soluciones eficientes y escalables a las problemáticas socioambientales.

- Los sujetos de investigación son investigadores y productores de conocimiento y no solo lo demandan y lo consumen; su participación activa, reflexiva y responsable en la producción de conocimiento los convierten en coinvestigadores y coproductores.

- La responsabilidad social se sustituye por procesos de empoderamiento de la comunidad. Ésta logra la comprensión de sus problemáticas y sus respectivas soluciones por medio de procesos autónomos pues adquieren los conocimientos, aptitudes y comportamientos para actuar en coherencia con la comprensión alcanzada de sus propias problemáticas en un proceso consciente y reflexivo.

- La validación es un proceso colectivo que se produce mediante la utilidad y el poder transformador que alcance el conocimiento producido en la comunidad. Se establecen redes de comunicación que se pueden mantener a través del tiempo y que se reconstruyen con la posibilidad de crear comunidades de práctica.

- Plantea una crítica a los modos de producción existentes y se convierte en una alternativa innovadora para la solución de problemas socioambientales. Pero asimismo es una crítica a un tipo de conocimiento denominado «científico» que se quiere implantar en la escuela como fin último de la educación.

- Permite superar la visión unidireccional del desarrollo y la visión simplista de la realidad para pasar a comprender la complejidad de las construcciones sociales, políticas, ambientales, económicas y culturales de la realidad de la humanidad. 
El modo 3 de producción de conocimiento implica para este trabajo investigativo la generación de una teoría didáctica para la formación de profesores que acompañen procesos de EA, así como conocimiento en torno a la construcción de conocimiento escolar deseable —en los alumnos y alumnas de bachillerato-que aporte a la comprensión de los procesos de enseñanza y aprendizaje de EA y que puedan escalarse a través de diferentes ciclos de investigación-acción, teniendo en cuenta los planteamiento de Elliot (2000).

Estas cuatro perspectivas teóricas pueden ser un marco referencial que apoyen el desarrollo tanto la investigación como la enseñanza y aprendizaje de la EA en las escuelas puesto que ésta requiere de la comprensión compleja de la realidad: las problemáticas socioambientales que solo pueden solucionarse desde diferentes perspectivas, tipos de conocimientos y esferas de la sociedad, cuestiones que se complementan adecuadamente con las características de los cuatro paradigmas explicados. Trabajos de investigación e implementación de estrategias didácticas de este corte pueden aportar aprendizajes significativos en el alumnado de bachillerato según experiencias referenciadas por el grupo del doctorado en Educación Ambiental en Sevilla, España.

A continuación se expondrán algunos aspectos de una propuesta en EA desde el PRAE de la IED Ignacio Pescador en la que se pretende desarrollar el currículo de EA a través de la interdisciplinariedad y la transversalidad.

\section{De la teoría a la práctica}

Para llevar a la práctica las perspectivas teóricas analizadas anteriormente, la IED Ignacio Pescador del municipio de Choachí - Cundinamarca ha implementado una propuesta de formación integral de los estudiantes a través del Proyecto Ambiental Escolar —PRAE— «Reconociendo nuestro territorio chiguano», desarrollado durante los últimos años, con el objetivo de formar a los estudiantes, docentes y padres de familia en competencias ciudadanas y comportamientos proambientales que contribuyan a la conservación del medio ambiente y generar ideas emprendedoras para la solución de problemáticas o el mejoramiento de la prestación del servicio ecoturístico, uno de los principales renglones económicos del municipio.

El marco conceptual del PRAE está inmerso en las perspectivas analizadas y es por ello que se pretende integrar el conocimiento cotidiano de la comunidad con el conocimiento científico dentro de las acciones pedagógicas en el aula; se conforma de este modo el conocimiento escolar deseable, el cual logra ser transferido al entorno mediante el análisis de diferentes factores relacionados con el medio ambiente y su relación con las asignaturas de la escuela. 
Las acciones pedagógicas del PRAE intervienen en diferentes dimensiones del ser humano y está dirigido a los estudiantes, docentes y familias de los grados sexto a undécimo de la institución. La principal actividad es la salida de campo a un sitio del municipio para reconocer sus características físicas, demográficas, geográficas, sociales, históricas y ambientales que a su vez son la excusa perfecta para desarrollar competencias ciudadanas relacionadas con el trabajo en equipo y mejorar las relaciones familiares y comunitarias entre sus miembros. Esto es así pues los estudiantes deben desarrollar trabajos de diferentes áreas del conocimiento en colaboración con sus pares pero, asimismo, con los padres de familia que los acompañan; también realizan labores de acompañamiento, liderazgo comunitario y aprendizaje.

De este modo profesores de diferentes áreas del conocimiento se integran al proyecto por medio del análisis de temáticas y trabajos relacionados con el entorno, lugares que se visitan dentro del paradigma de la complejidad, y la abolición de la dicotomía entre los diferentes tipos de conocimiento y entre la práctica y la teoría. Durante los dos años del proyecto se han logrado integrar áreas del conocimiento como inglés, emprendimiento, tecnología, ciencias naturales, sociales y educación física.

De otra parte, el reconocimiento del territorio a través de las salidas de campo y los trabajos de las distintas asignaturas ha permitido una visión local, compleja y transversal de la realidad del municipio: no solamente se observan los aspectos de la naturaleza y su biodiversidad, sino las características geográficas y físicas del entorno y las características de la comunidad. Se han realizado encuestas con los habitantes del lugar para conocer características socioeconómicas, modos de pensar y de actuar ante algunos recursos como el agua, se han analizado modos de vida teniendo en cuenta aspectos como las viviendas y cultivos y las posibilidades de empresas o negocios alrededor del agroturismo.

Un aspecto que ha sido importante en la implementación de este proyecto ha sido la participación de padres de familia y acudientes, quienes se empoderan de su rol como líderes del grupo de estudiantes y trabajan hombro a hombro con ellos; al mismo tiempo se capacitan en conocimientos ambientales y se admiran de los recursos naturales de su propio entorno dentro de la educación ambiental que se pretende realizar a partir de la institución educativa. Esta convivencia entre padres e hijos ha sido enriquecedora pues se conocen como comunidad educativa, comparten con los compañeros(as) de sus hijos(as) y se han estrechado lazos familiares y comunitarios.

La metodología que se implementa se relaciona con la investigación-acción como un ciclo que desde las fases de planeación, acción, observación y reflexión permite ir puliendo las debilidades, magnificar las fortalezas, aprender de los errores, 
aprovechar las oportunidades y buscar solución a las amenazas que día a día se pueden presentar. Es por ello que la reflexión continua sobre las acciones realizadas es el insumo principal para realimentar el proyecto para el siguiente año.

Finalmente, se crea conocimiento desde la realidad cotidiana de los habitantes de Choachí, sus hábitos, costumbres, forma de vida y realidad socioambiental, para que desde un conocimiento local y complejo se logren mejorar aspectos de la misma comunidad.

\section{A manera de conclusión}

La puesta en escena de las perspectivas teóricas en el aula ha sido enriquecedora y es el principio para la elaboración de una teoría acerca de los procesos de enseñanza y aprendizaje de la EA que puede establecer diferentes dinámicas y estrategias metodológicas para buscar el objetivo último de la educación: la solución en comunidad de sus problemas socioambientales para mejorar su calidad de vida.

En este caso ha sido gratificante el desarrollo de acciones pedagógicas que han transformado conocimientos cotidianos en conocimientos más elaborados, menos simplificados y más complejos, no solamente para los estudiantes, sino también para los padres de familia que aseguran la transferencia del conocimiento escolar fuera de sus paredes estructurales. La reflexión continua de las acciones realizadas ha permitido replantear tareas, conceptos y temas en diferentes áreas del conocimiento en los que se transforman conocimientos. Se ha logrado el reconocimiento de los ecosistemas chiguanos y la admiración de los participantes por el ambiente, procesos que pueden convertirse en una acción proambiental, importante para paliar la actual crisis ambiental. 


\section{Referencias}

Acosta, W., \& Carreño, C. (2013). Modo 3 de producción de conocimiento: implicaciones para la universidad de hoy. Revista de la Universidad de la Salle, 67-88.

Arroyo, J. (2012). La construcción del conocimiento sobre la gestión y la contaminación del agua. Concepciones del alumnado de primero de bachillerato (tesis doctoral). Sevilla: Universidad de Sevilla.

Cubero, R. (2000). Cómo trabajar con las ideas de los alumnos. Sevilla: Diada Editores.

Elliot, J. (2000). La investigación-acción en educación. Recuperado el 17 de abril de 2014, de http://plataforma.upnmazatlan.com/sites/default/files/180/ELLIOT

Fernández, J. (2012). La construcción del conocimiento sobre la gestión y la contaminación del agua. Concepciones del alumnado de primero de bachillerato (tesis doctoral). Sevilla: Universidad de Sevilla.

García Pérez, F. (s.f.). El modelo didáctico alternativo para transformar la educación: el modelo de investigación en la escuela. Recuperado el 17 de abril de 2014, de https://dialnet. unirioja.es/servlet/articulo?codigo=244386

García, J. (1998). Hacia una teoría alternativa sobre los contenidos escolares. Sevilla: Diada Editora.

García, J., \& Cano, M. (2006). ¿Cómo nos puede ayudar la perspectiva constructivista a construir conocimiento en Educación Ambiental? Revista Iberoamericana de Educación, 117-131.

García, M. (2010). El modelo sistémico complejo como instrumento de análisis del grado de complejidad de las actividades del campo de aprendizaje de los monasterios de Cister . En Doctorado Interuniversitario de Educación Ambiental, Investigar para avanzar en educación ambiental (pp. 102-120). Barcelona: Organismo Autónomo Parques Nacionales.

Kember, D., \& Lyngow. (1992). Action research as a form of staff development in Higher Education. HigherEducation, 297-310.

Recibido: 8 de febrero 2016

Aceptado: 24 de marzo 2016

Cómo citar: Gutiérrez S., L. (2016). Perspectivas teóricas para la

Educación Ambiental en básica secundaria. Teoría y práctica. Praxis

Pedagógica, 19, 61 - 75. 